\title{
Impact of absorption mechanisms on Kerr-nonlinear resonator behavior
}

\author{
Gino Priem, ${ }^{\text {a) }}$ Peter Bienstman, Geert Morthier, and Roel Baets \\ Department of Information Technology (INTEC), Ghent University, Sint-Pietersnieuwstraat 41, \\ 9000 Ghent, Belgium
}

(Received 26 May 2005; accepted 7 February 2006; published online 28 March 2006)

\begin{abstract}
The effect of both single-photon and two-photon absorption mechanisms on the Kerr-nonlinear behavior of resonant structures is investigated using a semianalytical, one-dimensional model. In particular, two-photon effects may severely degrade the ultrafast, nonlinear potential of these structures. Based on this model, the feasibility of Kerr-nonlinear operation is derived for the AlGaAs and Si material systems. (C) 2006 American Institute of Physics. [DOI: 10.1063/1.2184432]
\end{abstract}

\section{INTRODUCTION}

Nonlinear optics is known to have great potential for all-optical signal processing because of its intrinsically ultrafast response times. The practical use of effects such as the Kerr effect is, however, severely limited by the need for high optical power or long device lengths, as these effects are typically very small in common semiconductor systems $\left(n_{2}\right.$ $\approx 10^{-15}-10^{-13} \mathrm{~cm}^{2} / \mathrm{W}$ ).

This problem can be solved by using structures which show resonant behavior. In these resonant structures, the electric field is enhanced and the pulse is slowed down, so that the nonlinear response is larger. It has been shown that these components exhibit large improvements for the purpose of Kerr-nonlinear phase shifting. ${ }^{1-8}$ In addition, they also show features which cannot be implemented with plain waveguides, such as all-optical limiting, ${ }^{1,9}$ all-optical switching, and bistability. ${ }^{1,10-15}$ This enhancement of the nonlinearity, however, happens at the cost of reduction of the signal bandwidth, which typically leads to a trade-off situation. ${ }^{1,2}$

In practice, however, resonant structures such as ring resonators and photonic crystal resonators are much more critical to fabricate than simple waveguides and therefore suffer from additional loss mechanisms such as radiation and scattering loss. Furthermore, two-photon absorption (2PA) — as intrinsic counterpart of the Kerr effect-will also be present: in these resonant structures, the two-photon absorption effect is enhanced by the same principle-optical confinement and slow waving_as the Kerr effect. ${ }^{5,16}$ The impact of these two absorption effects on resonant Kerrnonlinear behavior will be investigated in this paper in the case of a single resonator in a semianalytical way. In this way, their impact can rapidly be determined for different materials systems and configurations.

The organization of this paper is as follows. First, the lossless resonator behavior discussed in Refs. 1 and 2 will be reviewed. After that, the effect of single-photon absorption and two-photon absorption is discussed in Secs. III and IV. The combination of these two effects together is then studied in Sec. V. In Sec. VI, the feasibility of Kerr-nonlinear operation in the presence of both single-photon and two-photon absorptions is derived for two material systems: AlGaAs and Si. Finally, conclusions are drawn in Sec. VII.

\section{LOSSLESS KERR-NONLINEAR RESONATOR OPERATION}

In this section, the most important equations from Refs. 1 and 2 are summarized. A resonator structure can generally be understood as a cavity which is placed between two mirrors. In this work, the same one-dimensional resonator structures will be discussed as in Refs. 1 and 2: the mirror sections are built from quarter-wavelength layers of two different refractive index materials $n_{a}$ and $n_{b}$, and the cavity is assumed to be a multiple $N_{\text {cav }}$ of $\lambda_{c} / 2 n_{a}$, with $\lambda_{c}$ the so called resonance wavelength (see below), so that the resonator structure is schematically given by ${ }^{1}$

$$
\underbrace{a_{\mathrm{in}}}_{\text {input }} \underbrace{b_{\lambda_{c} / 4}\left(a_{\lambda_{c} / 4} b_{\lambda_{c} / 4}\right)^{\left(N_{\mathrm{dbr}} / 2\right)-1}}_{\text {mirror }} \underbrace{a_{N_{\text {cav }}\left(\lambda_{c} / 2\right)}}_{\text {cavity }} \underbrace{b_{\lambda^{\prime 4}}\left(a_{\lambda_{c} / 4} b_{\lambda_{c} / 4}\right)^{\left(N_{\mathrm{dbr}} / 2\right)-1}}_{\text {mirror }} \underbrace{a_{\text {out }}}_{\text {output }}
$$

$N_{\mathrm{dbr}}$ is the total number of b layers, and the input and output sections were assumed to have the same effective index as the cavity. An example of such a structure is shown in Fig. 1.

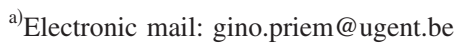

\section{A. Linear behavior}

The cavity of a resonator typically gives rise to a phase change $e^{-j \varphi_{\text {cav }}}$ for the light passing through, while the mirrors (which are assumed to be equal) cause both phase and amplitude changes. As a result, the mirror amplitude transmis- 


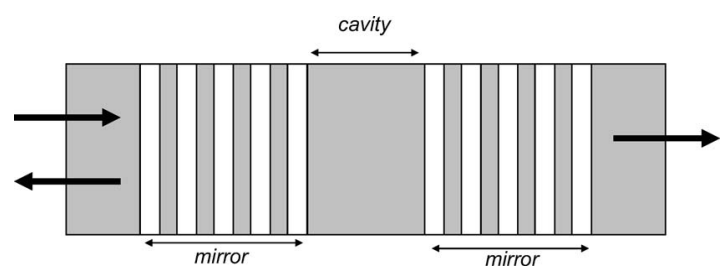

FIG. 1. Structural example of a single resonator. Mirror sections and cavity are indicated.

sion and reflection coefficients $t_{\mathrm{dbr}}$ and $r_{\mathrm{dbr}}$ can formally be written as

$$
\begin{aligned}
& t_{\mathrm{dbr}}=\left|t_{\mathrm{dbr}}\right| e^{-j \varphi_{t},} \\
& r_{\mathrm{dbr}}=\left|r_{\mathrm{dbr}}\right| e^{-j \varphi_{r},},
\end{aligned}
$$

with $\left|t_{\mathrm{dbr}}\right|^{2}+\left|r_{\mathrm{dbr}}\right|^{2}=1$ in the lossless case.

The linear amplitude transfer function of a single resonator can be calculated by adding up the different wave contributions which circulate inside the structure, resulting in

$$
\begin{aligned}
t_{\mathrm{tot}, \mathrm{L}}(\nu)= & \left.e^{-j \varphi_{\mathrm{cav}} \mid} t_{\mathrm{dbr}}\right|^{2} e^{-2 j \varphi_{t}}\left(1+e^{-2 j \varphi_{\mathrm{cav}}}\left|r_{\mathrm{dbr}}\right|^{2} e^{-2 j \varphi_{r}}\right. \\
& +e^{\left.-4 j \varphi_{\mathrm{cav}}\left|r_{\mathrm{dbr}}\right|^{4} e^{-4 j \varphi_{r}}+\cdots\right),} \\
= & e^{-j \varphi_{\mathrm{cav}}} \frac{\left|t_{\mathrm{dbr}}\right|^{2} e^{-2 j \varphi_{t}}}{1-e^{-2 j \varphi_{r}-2 j \varphi_{\mathrm{cav}}}\left|r_{\mathrm{dbr}}\right|^{2}} .
\end{aligned}
$$

The resulting linear intensity transmission function is then given by

$$
\left|t_{\mathrm{tot}, \mathrm{L}}(\nu)\right|^{2}=\frac{\left|t_{\mathrm{dbr}}\right|^{4}}{1+\left|r_{\mathrm{dbr}}\right|^{2}-2\left|r_{\mathrm{dbr}}\right|^{2} \cos \left(2 \varphi+2 \varphi_{\mathrm{cav}}\right)} .
$$

This transmission is equal to unity if $\varphi_{r}+\varphi_{\text {cav }}=m \pi$ with $m$ integer. Frequencies for which $\varphi_{r}+\varphi_{\text {cav }}=m \pi$ are called the resonance frequencies and denoted as $\nu_{c}$.

If the frequency dependence of the amplitude of the mirror transmission and reflection coefficients $t_{\mathrm{dbr}}$ and $r_{\mathrm{dbr}}$ is neglected and the frequency dependence of the mirror phase is expanded linearly $\left|t_{\text {tot, } \mathrm{L}}(\nu)\right|^{2}$ can also be written as

$$
\left|t_{\mathrm{tot}, \mathrm{L}}(\nu)\right|^{2}=\frac{\left|t_{\mathrm{dbr}}\right|_{\nu_{c}}^{4}}{1+\left|r_{\mathrm{dbr}}\right|_{\nu_{c}}^{4}-2\left|r_{\mathrm{dbr}}\right|_{\nu_{c}}^{2} \cos \left[U\left(\nu-\nu_{c}\right)\right]},
$$

taking into account that $\varphi_{\text {cav }} \propto \nu$ and $U$ is constant. Close to resonance $\left(\nu \approx \nu_{c}\right)$, one obtains the lorentz form

$$
\left|t_{\mathrm{tot}, \mathrm{L}}(\nu)\right|^{2}=\frac{1}{1+\left|r_{\mathrm{dbr}}\right|_{\nu_{c}}^{2} U^{2}\left(\nu-\nu_{c}\right)^{2}},
$$

so that $U=2 /\left|r_{\mathrm{dbr}}\right|_{c} \Delta \nu_{\mathrm{BW}, \text { lossless }}$, with $\Delta \nu_{\mathrm{BW}, \text { lossless }}$ the lossless resonance bandwidth.

\section{B. Kerr-nonlinear behavior}

In the presence of Kerr-nonlinear effects, the incoming light with electric field amplitude $E_{\text {in }}$ will build up inside the cavity and partially in the mirrors and thus alter the refractive index of the complete structure by an amount $\Delta n=n_{2}|E|^{2}\left(n_{2}\right.$ being the Kerr coefficient and $E$ the electric field). As a re- sult, the linear resonance frequency $\nu_{c}$ will shift by an amount $\Delta \nu_{c \text {,lossless }}$ (this shift is negative if $n_{2}>0$ and vice versa). The frequency shift for the frequency $\nu \neq \nu_{c}$ will, however, not be equal to $\Delta \nu_{c}$. The field profile of a resonator for a certain frequency $\nu$ scales, in good approximation, with a factor $\left|t_{\text {tot, } \mathrm{L}}(\nu)\right|$ compared to that for the frequency $\nu_{c}{ }^{1}$ As the Kerr effect scales with $|E|^{2}$, the shift at frequency $\nu$ will only be

$$
\Delta \nu_{\mathrm{NL}} \approx\left|t_{\mathrm{tot}, \mathrm{NL}}(\nu)\right|^{2} \Delta \nu_{c, \text { lossless }}
$$

which means that the Kerr-nonlinear intensity transmission for one period is determined by

$$
\left|t_{\text {tot, NL }}\left(\nu^{\prime}\right)\right|^{2}=\left|t_{\text {tot, } \mathrm{L}}(\nu)\right|^{2},
$$

with $\nu^{\prime}=\nu+\left|t_{\mathrm{tot}, \mathrm{L}}(\nu)\right|^{2} \Delta \nu_{c}$. Using Eq. (7), this corresponds to

$$
\begin{aligned}
\mid t_{\mathrm{tot}, \mathrm{NL}} & \left.\left(\nu^{\prime}\right)\right|^{6}-2 \frac{\nu^{\prime}-\nu_{c}}{\Delta \nu_{c, \text { lossless }}}\left|t_{\mathrm{tot}, \mathrm{NL}}\left(\nu^{\prime}\right)\right|^{4} \\
& +\left[\frac{\Delta \nu_{\mathrm{BW}, \text { lossless }}^{2}}{4 \Delta \nu_{c, \text { lossless }}^{2}}+\left(\frac{\nu^{\prime}-\nu_{c}}{\Delta \nu_{c, \text { lossless }}}\right)^{2}\right]\left|t_{\mathrm{tot}, \mathrm{NL}}\left(\nu^{\prime}\right)\right|^{2} \\
& -\frac{\Delta \nu_{\mathrm{BW}, \text { lossless }}^{2}}{4 \Delta \nu_{c, \text { lossless }}^{2}}=0 .
\end{aligned}
$$

This is an equation of the third order in $\left|t_{\text {tot,NL }}\left(\nu^{\prime}\right)\right|^{2}$ and formally identical to the one derived in Ref. 17. Since its coefficients are real for every frequency, one solution is always real and the other two solutions can be either complex conjugants or also real. In the latter case, one has three possible solutions for the same frequency, of which only two are stable-the solution is said to be bistable. In such a case, $\left|t_{\text {tot,NL }}\left(\nu^{\prime}\right)\right|^{2}$ is not anymore a "function" in the strict sense of the word.

A bistable region now exist, if for two frequencies $\nu_{1}$ and $\nu_{2}$, with $\nu_{1}>\nu_{2}$, one has that $\nu_{1}^{\prime}<\nu_{2}^{\prime}$, with $\nu^{\prime}=\nu$ $+\left|t_{\text {tot }, \mathrm{L}}(\nu)\right|^{2} \Delta \nu_{c, \text { lossless }}=\nu+\left|t_{\text {tot }, \mathrm{NL}}\left(\nu^{\prime}\right)\right|^{2} \Delta \nu_{c \text {, lossless }}$. For the case $n_{2}>0$, this results in the following condition:

$$
\exists \nu, \quad 0<-\left[\frac{d\left|t_{\mathrm{tot}, \mathrm{L}}(\nu)\right|^{2}}{d \nu}\right]^{-1}<\Delta \nu_{c, \text { lossless }} .
$$

The bistable region can then be obtained by solving

$$
\left[\frac{d\left|t_{\mathrm{tot}, \mathrm{L}}(\nu)\right|^{2}}{d \nu}\right]^{-1}=-\Delta_{c, \text { lossless }}
$$

and calculating $\nu^{\prime}=\nu+\left|t_{\text {tot, } \mathrm{L}}(\nu)\right|^{2} \Delta \nu_{c \text {, lossless }}$.

In this way, it can also be determined from which amount of resonance shift $\Delta \nu_{c}$,lossless a bistable region appears. This is the case if Eq. (12) has a twofold, real solution. Using Eq. (7), bistability arises if

$$
\left|\Delta \nu_{c, \text { lossless }}\right|>\frac{4 \sqrt{3}}{9} \Delta \nu_{\mathrm{BW}, \text { lossless }}
$$




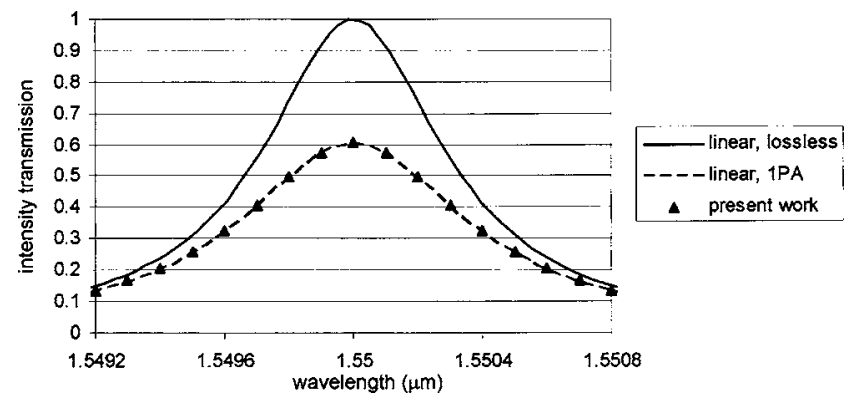

FIG. 2. Effect of single-photon absorption on the linear resonator behavior. The results were obtained by simulation with the following parameters: refractive indices $n_{a}=2.6, n_{b}=2.34$, extinction coefficients $k_{a}=k_{b}=0.15$ $\times 10^{-3}, N_{\text {cav }}=1$, and $N_{\mathrm{dbr}}=54$. The triangles show the analytical results for the lossy structure obtained by means of Eq. (17).

and the twofold solution is given by

$$
\nu_{\mathrm{sol}}=\nu_{c} \pm \frac{\sqrt{3}}{2} \Delta \nu_{\mathrm{BW}, \text { lossless }},
$$

depending on the sign of $\Delta \nu_{c}$. This also means that frequencies inside the interval $\left[\nu_{c}-(\sqrt{3} / 2) \Delta \nu_{\mathrm{BW}, \text { lossless }}, \nu_{c}\right.$ $\left.+(\sqrt{3} / 2) \Delta \nu_{\mathrm{BW}, \text { lossless }}\right]$ can in fact never be bistable.

\section{EFFECT OF SINGLE-PHOTON ABSORPTION (1PA)}

Single-photon absorption is not necessarily present in the case of Kerr-nonlinear behavior-especially not for typical wavelength operation around half the band gap of the material. ${ }^{18}$ In reality, however, different loss mechanisms will always be present, which have the appearance of a single-photon absorption term, such as scattering loss, radiation loss, etc.

These phenomena not only change the Kerr-nonlinear behavior of the structure, but also its linear behavior.

\section{A. Linear behavior}

First, the effect of single-photon absorption (or equivalent loss mechanisms) on the linear behavior of a resonator is studied. In Fig. 2, the simulated linear transmission relations of a single resonator are shown in the case of no loss and of loss due to 1PA.

1PA loss reduces the peak transmission, but it also changes the shape of the transmission relation (relative to the peak transmission) to a non-Lorentzian curve.
In the presence of single-photon absorption, the linear transfer function can be calculated in the same way as in the lossless case,

$$
\begin{aligned}
& t_{\mathrm{tot}, \mathrm{L}}(\nu)=e^{-j \varphi_{\mathrm{cav}}}\left|t_{\mathrm{dbr}}\right|_{\nu_{e}}^{2} e^{-2 j \varphi_{t}} A_{\mathrm{dbr}}^{2} A_{\mathrm{cav}}\left(1+e^{-2 j \varphi_{\mathrm{cav}}}\left|r_{\mathrm{dbr}}\right|_{\nu_{c}}^{2} e^{-2 j \varphi_{r}}\right. \\
& \left.\times A_{\mathrm{dbr}}^{2} A_{\mathrm{cav}}^{2}+\cdots\right), \\
& =e^{-j \varphi_{\mathrm{cav}}} \frac{\left|t_{\mathrm{dbr}}\right|_{\nu_{c}}^{2} e^{-2 j \varphi_{t}} A_{\mathrm{dbr}}^{2} A_{\mathrm{cav}}}{1-e^{-2 j \varphi_{r}-2 j \varphi_{\mathrm{cav}}}\left|r_{\mathrm{dbr}}\right|_{\nu_{c}}^{2} A_{\mathrm{dbr}}^{2} A_{\mathrm{cav}}^{2}},
\end{aligned}
$$

with $A_{\mathrm{dbr}}$ and $A_{\text {cav }}$ the relative field amplitude after a lossy mirror, respectively, after a lossy cavity section. For example, $A_{\text {cav }}=\exp (-(\alpha / 2) L)$, with $\alpha$ the single-photon absorption coefficient and $L$ the cavity length.

In this way, the intensity transmission curve (for $\nu \approx \nu_{c}$ ) is now given by

$$
\left|t_{\mathrm{tot}, \mathrm{L}}(\nu)\right|^{2} \approx \frac{\frac{\left|t_{\mathrm{dbr}}\right|_{\nu_{c}}^{4} A_{\mathrm{dbr}}^{4} A_{\mathrm{cav}}^{2}}{\left(1-\left|r_{\mathrm{dbr}}\right|_{\nu}^{2} A_{\mathrm{dbr}}^{2} A_{\mathrm{cav}}^{2}\right)^{2}}}{1+\frac{4\left|t_{\mathrm{dbr}}\right|_{\nu_{c}}^{4} A_{\mathrm{dbr}}^{2} A_{\mathrm{cav}}^{2}}{\left(1-\left|r_{\mathrm{dbr}}\right|_{\nu}^{2} A_{\mathrm{dbr}}^{2} A_{\mathrm{cav}}^{2}\right)^{2}} \frac{\left(\nu-\nu_{c}\right)^{2}}{\Delta \nu_{\mathrm{BW}, \text { lossless }}^{2}}} .
$$

This relation is used in Fig. 2 to explain the simulation results obtained with the simulation tool cavity modeling framework $^{19}$ (CAMFR). As can be seen, an excellent agreement with the numerical results is obtained.

At resonance, one has, for the total transmission,

$$
\left|t_{\mathrm{tot}, \mathrm{L}}\left(\nu_{c}\right)\right|^{2}=\frac{\left|t_{\mathrm{dbr}}\right|_{\nu_{c}}^{4} A_{\mathrm{dbr}}^{4} A_{\mathrm{cav}}^{2}}{\left(1-\left|r_{\mathrm{dbr}}\right|_{\nu}^{2} A_{\mathrm{dbr}}^{2} A_{\mathrm{cav}}^{2}\right)^{2}} \text {. }
$$

In a similar way, the total reflection $\left|r_{\text {tot,L }}(\nu)\right|^{2}$ can be calculated. The insertion loss $1-A_{\text {tot }}^{2}(\nu)$-with $A_{\text {tot }}(\nu)$ the relative field amplitude after the complete resonator structure in the case of linear loss-is then simply given by $1-\left|t_{\text {tot, } \mathrm{L}}(\nu)\right|^{2}$ $-\left|r_{\text {tot, } \mathrm{L}}(\nu)\right|^{2}$. At resonance, one has

$$
\begin{gathered}
\left|r_{\mathrm{tot}, \mathrm{L}}\left(\nu_{c}\right)\right|^{2}=\frac{\left|r_{\mathrm{dbr}}\right|_{\nu_{c}}^{2} A_{\mathrm{dbr}}^{2}\left(1-A_{\mathrm{dbr}}^{2} A_{\mathrm{cav}}^{2}\right)^{2}}{\left(1-\left|r_{\mathrm{dbr}}\right|_{\nu_{c}}^{2} A_{\mathrm{dbr}}^{2} A_{\mathrm{cav}}^{2}\right)^{2}}, \\
1-A_{\mathrm{tot}}^{2}\left(\nu_{c}\right)=1-\frac{\left|t_{\mathrm{dbr}}\right|_{\nu_{c}}^{4} A_{\mathrm{dbr}}^{4} A_{\mathrm{cav}}^{2}+\left|r_{\mathrm{dbr}}\right|_{\nu_{c}}^{2} A_{\mathrm{dbr}}^{2}\left(1-A_{\mathrm{dbr}}^{2} A_{\mathrm{cav}}^{2}\right)^{2}}{\left(1-\left|r_{\mathrm{dbr}}\right|_{\nu}^{2} A_{\mathrm{dbr}}^{2} A_{\mathrm{cav}}^{2}\right)^{2}} .
\end{gathered}
$$

For frequencies $\nu \neq \nu_{c}$, one has, in good approximation,

$$
1-A_{\mathrm{tot}}^{2}(\nu) \approx\left|t_{\mathrm{tot}}(\nu)\right|^{2}\left[\frac{\left(1-\left|r_{\mathrm{dbr}}\right|_{\nu}^{2} A_{\mathrm{dbr}}^{2} A_{\mathrm{cav}}^{2}\right)^{2}-\left|r_{\mathrm{dbr}}\right|_{\nu}^{2} A_{\mathrm{dbr}}^{2}\left(1-A_{\mathrm{dbr}}^{2} A_{\mathrm{cav}}^{2}\right)^{2}}{\left|t_{\mathrm{dbr}}\right|_{\nu}^{4} A_{\mathrm{dbr}}^{4} A_{\mathrm{cav}}^{2}}-1\right],
$$




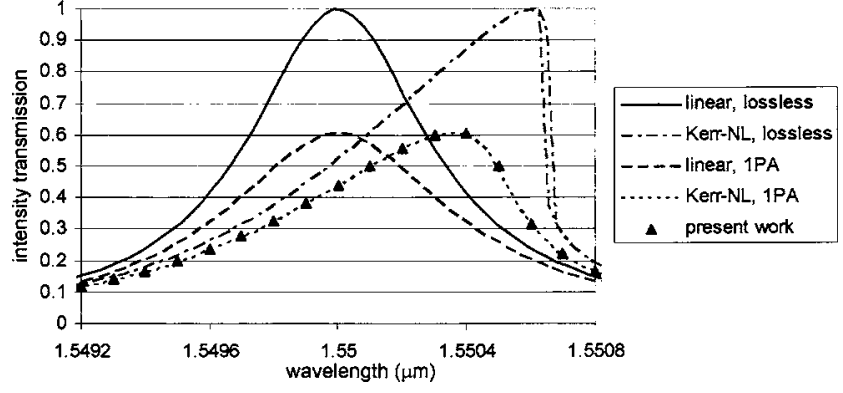

FIG. 3. Effect of single-photon absorption on the Kerr-nonlinear resonator behavior. The results were obtained by simulation with the following parameters: $n_{a}=2.6, n_{b}=2.34, k_{a}=k_{b}=0.15 \times 10^{-3}, N_{\text {cav }}=1, N_{\mathrm{dbr}}=54$, and $n_{2}\left|E_{\text {in }}\right|^{2}$ $=8 \times 10^{-6}$. The triangles show the analytical results for the lossy, nonlinear situation obtained by means of Eq. (24).

which means that the loss is in fact linearly dependent on the transmission.

\section{B. Kerr-nonlinear behavior}

Now that the change in the linear behavior of the resonator structure due to $1 \mathrm{PA}$ is understood, one can investigate the effect on its Kerr-nonlinear behavior. This is shown in Fig. 3. In this figure, both the linear and the Kerr-nonlinear transmission curves are shown for the lossless and 1PA induced lossy cases of the same resonator.

As can be seen, the resonance shift $\Delta \nu_{c}$ in the lossy case is significantly smaller than in the lossless case. Furthermore, in both cases, the nonlinear peak transmission remains equal to its linear counterpart.

As in Sec. II, the frequency shift $\Delta \nu$ can be approximated by

$$
\Delta \nu_{\mathrm{NL}}=\left|t_{\mathrm{tot}, \mathrm{L}}(\nu)\right|^{2} \Delta \nu_{c, \text { lossless }} .
$$

In the same way, the nonlinear transmission function can be determined by $\left|t_{\mathrm{tot}, \mathrm{NL}}\left(\nu^{\prime}\right)\right|^{2}=\left|t_{\mathrm{tot}, \mathrm{L}}(\nu)\right|^{2}$, with $\nu^{\prime}=\nu$ $+\left|t_{\text {tot }, \mathrm{L}}(\nu)\right|^{2} \Delta \nu_{c, \text { lossless }}=\nu+\left|t_{\text {tot }, \mathrm{NL}}\left(\nu^{\prime}\right)\right|^{2} \Delta \nu_{c, \text { lossless }}$. Substitution of Eq. (17) leads to

$$
\begin{gathered}
\left|t_{\text {tot,NL }}(\nu)\right|^{6}-\frac{2\left(\nu^{\prime}-\nu_{c}\right)}{\Delta \nu_{c, \text { lossless }}}\left|t_{\mathrm{tot}, \mathrm{NL}}\left(\nu^{\prime}\right)\right|^{4}+\left(\frac{\left(\nu^{\prime}-\nu_{c}\right)^{2}}{\Delta \nu_{c, \text { lossless }}^{2}}\right. \\
\left.+\frac{\Delta \nu_{\mathrm{BW}, \text { lossless }}^{2}}{4 \Delta \nu_{c, \text { lossless }}^{2}} \frac{\left(1-\left|r_{\mathrm{dbr}}\right|_{\nu_{c}}^{2} A_{\mathrm{dbr}}^{2} A_{\mathrm{cav}}^{2}\right)^{2}}{\left|t_{\mathrm{dbr}}\right|_{\nu_{c}}^{4} A_{\mathrm{dbr}}^{2} A_{\mathrm{cav}}^{2}}\right)\left|t_{\mathrm{tot}, \mathrm{NL}}\left(\nu^{\prime}\right)\right|^{2} \\
=\left|t_{\mathrm{tot}, \mathrm{L}}\left(\nu_{c}\right)\right|^{2} \frac{\Delta \nu_{\mathrm{BW}, \text { lossless }}^{2}}{4 \Delta \nu_{c, \text { lossless }}^{2}} \frac{\left(1-\left|r_{\mathrm{dbr}}\right|_{\nu_{c}}^{2} A_{\mathrm{dbr}}^{2} A_{c a v}^{2}\right)^{2}}{\left|t_{\mathrm{dbr}}\right|_{\nu_{c}}^{4} A_{\mathrm{dbr}}^{2} A_{\mathrm{cav}}^{2}}
\end{gathered}
$$

which approximately simplifies to

$$
\begin{gathered}
\left|t_{\mathrm{tot}, \mathrm{NL}}\left(\nu^{\prime}\right)\right|^{6}-\frac{2\left(\nu^{\prime}-\nu_{c}\right)}{\Delta_{c, \text { lossless }}}\left|t_{\mathrm{tot}, \mathrm{NL}}\left(\nu^{\prime}\right)\right|^{4}+\left[\frac{\left(\nu^{\prime}-\nu_{c}\right)^{2}}{\Delta \nu_{c, \text { lossless }}^{2}}\right. \\
\left.+\frac{\Delta \nu_{\mathrm{BW}, \text { lossless }}^{2}}{4\left|t_{\mathrm{tot}}\left(\nu_{c}\right)\right|^{2} \Delta \nu_{c, \text { lossless }}^{2}}\right]\left|t_{\mathrm{tot}, \mathrm{NL}}\left(\nu^{\prime}\right)\right|^{2}=\frac{\Delta \nu_{\mathrm{BW}, \text { lossless }}^{2}}{4 \Delta \nu_{c, \text { lossless }}^{2}}
\end{gathered}
$$

The analytical results obtained by this equation show excel-

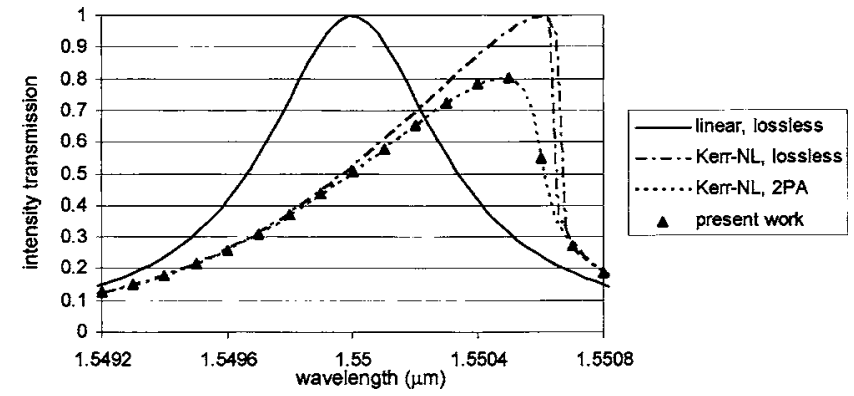

FIG. 4. Effect of two-photon absorption on the Kerr-nonlinear resonator behavior. The results were obtained by simulation with the following parameters: $n_{a}=2.6, n_{b}=2.34, N_{\text {cav }}=1, N_{\mathrm{dbr}}=54$, and $n_{2}\left|E_{\mathrm{in}}\right|^{2}=\beta \lambda_{c}\left|E_{\mathrm{in}}\right|^{2}=8 \times 10^{-6}$. The triangles show the analytical results for the lossy, nonlinear situation obtained by means of Eq. (46).

lent agreement with the nonlinear numerical results obtained by the nonlinear extension ${ }^{20}$ to CAMFR. ${ }^{19}$

\section{EFFECT OF TWO-PHOTON ABSORPTION (2PA)}

As mentioned above, the bound-electronic Kerr effect is intrinsically related to two-photon absorption. This absorption effect is enhanced inside the resonator in the same way as the Kerr-nonlinear effect and thus poses an inherent limitation to its applicability.

In Fig. 4, an example of the effect of two-photon absorption is given. The linear transmission curve and both the lossless and lossy (due to 2PA) frequency shifts obtained by simulations are shown.

As can be seen, the resonance shift $\Delta \nu_{\mathrm{NL}}$ in the lossy case is again significantly smaller than in the lossless case. The lossy, nonlinear peak transmission is also smaller than the linear one, as discussed in Sec. II. In addition, the lossy, Kerr-nonlinear transmission relation has a different relative shape than its lossless counterpart. This will now be investigated in the following way: similar to Sec. III, the total insertion loss and the resonance shift will first be determined at resonance and then for a general frequency $\nu$. However, in the general case of counterpropagating waves, the multitime-scale approach used in Refs. 1 and 2 for the Kerr effect cannot be used to study the effect of 2PA. Therefore, simulation results from different types of resonator structures (both long and short cavities combined with weak and strong mirror sections) will be used to characterize the influence of two-photon absorption in a general way. With these results, an equation for $\left|t_{\mathrm{tot}, \mathrm{NL}}\left(\nu^{\prime}\right)\right|^{2}$ will then be determined equivalent to Eq. (24).

The total transmission, reflection, and loss of any structure are for every frequency related by the following equation:

$$
\left|t_{\mathrm{tot}}(\nu)\right|^{2}+\left|r_{\mathrm{tot}}(\nu)\right|^{2}=B_{\mathrm{tot}}^{2}(\nu),
$$

with $t_{\text {tot }}(\nu)$ and $r_{\text {tot }}(\nu)$ the total (nonlinear) field transmission and reflection, respectively, and $B_{\text {tot }}(\nu)$ the relative field amplitude after the complete resonator structure in the case of two-photon absorption. Two additional relations between $\left|t_{\text {tot }}(\nu)\right|^{2},\left|r_{\text {tot }}(\nu)\right|^{2}$, and $B_{\text {tot }}^{2}(\nu)$ now have to be found to determine these three quantities completely. 


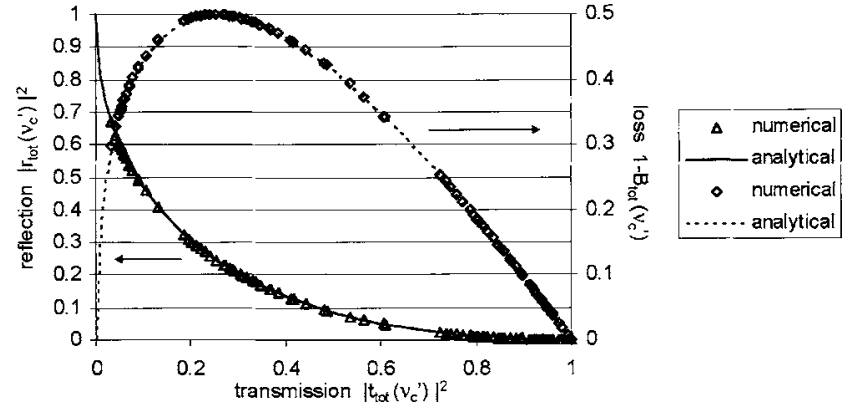

FIG. 5. Simulated results of $\left|r_{\text {tot }}\left(\nu_{c}^{\prime}\right)\right|^{2}$ and 1- $B_{\text {tot }}\left(\nu_{c}^{\prime}\right)$ as a function of $\left|t_{\text {tot }}\left(\nu_{c}^{\prime}\right)\right|^{2}$ for a variety of parameters.

At nonlinear resonance $\left(\nu=\nu_{c}^{\prime}\right.$, with $\nu_{c}^{\prime}$ the nonlinear resonance frequency), this can be done by fitting the simulation results presented in Fig. 5. In this figure, the simulation results $\left|r_{\text {tot }}\left(\nu_{c}^{\prime}\right)\right|^{2}$ and $1-B_{\text {tot }}\left(\nu_{c}^{\prime}\right)$ are plotted as a function of $\left|t_{\text {tot }}\left(\nu_{c}^{\prime}\right)\right|^{2}$ for a large variation of all resonator parameters: the refractive indices $\left(n_{a}\right.$ and $\left.n_{b}\right)$ of the two mirror materials were varied from 2.0-3.0, with $n_{a}>n_{b}$. Mirror and cavity lengths in the intervals $N_{\mathrm{dbr}}=40-200$ and $N_{\text {cav }}=1-20$ were simulated with input fields leading to nonlinear effects of $n_{2}\left|E_{\text {in }}\right|^{2}=10^{-6}-10^{-4}$ and $\beta \lambda_{c}\left|E_{\text {in }}\right|^{2}=10^{-6}-10^{-4}$, with $\beta$ the two-photon absorption coefficient and $\lambda_{c}=1.55 \mu \mathrm{m}$.

It can be seen that the relation between $\left|r_{\text {tot }}\left(\nu_{c}^{\prime}\right)\right|^{2}$ and $\left|t_{\text {tot }}\left(\nu_{c}^{\prime}\right)\right|^{2}$ is symmetrical around $\left|r_{\text {tot }}\left(\nu_{c}^{\prime}\right)\right|^{2}=\left|t_{\text {tot }}\left(\nu_{c}^{\prime}\right)\right|^{2}$. This relation can therefore be expressed as

$$
\begin{gathered}
p+q\left(\left|t_{\mathrm{tot}}\left(\nu_{c}^{\prime}\right)\right|^{2}+\left|r_{\mathrm{tot}}\left(\nu_{c}^{\prime}\right)\right|^{2}\right)+r\left|t_{\mathrm{tot}}\left(\nu_{c}^{\prime}\right)\right|^{2}\left|r_{\mathrm{tot}}\left(\nu_{c}^{\prime}\right)\right|^{2} \\
+s\left(\left|t_{\mathrm{tot}}\left(\nu_{c}^{\prime}\right)\right|^{4}+\left|r_{\mathrm{tot}}\left(\nu_{c}^{\prime}\right)\right|^{4}\right)+\cdots=0 .
\end{gathered}
$$

It was found that the choice

$$
\begin{aligned}
& p=1, \\
& q=-2, \\
& r=-2, \\
& s=1,
\end{aligned}
$$

holds a very good approximation for the obtained simulation results (see Fig. 5). In this way, one has

$\left[\left|t_{\text {tot }}\left(\nu_{c}^{\prime}\right)\right|^{2}-\left|r_{\text {tot }}\left(\nu_{c}^{\prime}\right)\right|^{2}\right]^{2}-2\left[\left|t_{\text {tot }}\left(\nu_{c}^{\prime}\right)\right|^{2}+\left|r_{\text {tot }}\left(\nu_{c}^{\prime}\right)\right|^{2}\right]+1 \approx 0$.

As all parameters used in the simulations were varied over a large range, this relation will be generally valid. Now, both the (nonlinear) resonance transmission and reflection can be expressed in terms of $B_{\mathrm{tot}}^{2}\left(\nu_{c}^{\prime}\right)$ as

$$
\begin{aligned}
& \left|t_{\text {tot }}\left(\nu_{c}^{\prime}\right)\right|^{2}=\frac{1}{2}\left[B_{\text {tot }}^{2}\left(\nu_{c}^{\prime}\right)+\sqrt{2 B_{\text {tot }}^{2}\left(\nu_{c}^{\prime}\right)-1}\right], \\
& \left|t_{\text {tot }}\left(\nu_{c}^{\prime}\right)\right|^{2}=\frac{1}{2}\left[B_{\text {tot }}^{2}\left(\nu_{c}^{\prime}\right)-\sqrt{2 B_{\text {tot }}^{2}\left(\nu_{c}^{\prime}\right)-1}\right] .
\end{aligned}
$$

To determine this nonlinear loss, the following method is used. For small intensities, the loss due to two-photon absorption is equivalent to the phase shift at resonance induced by the Kerr effect,

$$
1-B_{\mathrm{tot}, \mathrm{small}}^{2}\left(\nu_{c}^{\prime}\right) \propto 2 \frac{\omega K_{2}}{c}\left|E_{\mathrm{in}}\right|^{2} \Leftrightarrow \Delta \phi_{c} \propto-\frac{\omega n_{2}}{c}\left|E_{\mathrm{in}}\right|^{2},
$$

with $K_{2}=(c / 4 \pi \nu) \beta$ the nonlinear extinction coefficient and $\beta$ the two-photon absorption coefficient. This relation represents nothing more than the refractive index-absorption coefficient duality. The factor 2 is due to the fact that loss is related to the optical intensity, whereas the phase is related to the optical field. The lossless resonance phase shift $\Delta \phi_{c, \text { lossless }}$ is related to the resonance shift $\Delta \nu_{c, \text { lossless }}$ by ${ }^{1,2}$

$$
\Delta \phi_{c, \text { lossless }} \approx \frac{2}{\Delta \nu_{\mathrm{BW}, \text { lossless }}} \Delta \nu_{c, \text { lossless }} \text {. }
$$

In this way, one has, for small intensities,

$$
1-B_{\text {tot, } \text {,small }}^{2}\left(\nu_{c}^{\prime}\right)=-\frac{c}{\pi \nu_{c}^{\prime}} \frac{\beta}{n_{2}} \frac{\Delta \nu_{c, \text { lossless }}}{\Delta \nu_{\mathrm{BW}, \text { lossless }}} .
$$

For the general case, it was found by fitting the results of Fig. 5,

$$
\begin{aligned}
1-B_{\mathrm{tot}}^{2}\left(\nu_{c}^{\prime}\right) & \approx\left|t_{\mathrm{tot}, \mathrm{NL}}\left(\nu_{c}^{\prime}\right)\right|^{4}\left[1-B_{\mathrm{tot}, \text { small }}^{2}\left(\nu_{c}^{\prime}\right)\right] \\
& =-\left|t_{\mathrm{tot}}\left(\nu_{c}^{\prime}\right)\right|^{4} \frac{c}{\pi \nu_{c}^{\prime}} \frac{\beta}{n_{2}} \frac{\Delta \nu_{c, \text { lossless }}}{\Delta \nu_{\mathrm{BW}, \text { lossless }}} .
\end{aligned}
$$

This $\left|t_{\text {tot,NL }}\left(\nu_{c}^{\prime}\right)\right|^{4}$ is at first unexpected, but will be explained below. Using Eq. (32), one obtains the following implicit formula for the insertion loss:

$$
\begin{aligned}
1-B_{\mathrm{tot}}^{2}\left(\nu_{c}^{\prime}\right) \approx & -\frac{c}{4 \pi \nu_{c}^{\prime}} \frac{\beta}{n_{2}}\left[B_{\mathrm{tot}}^{2}\left(\nu_{c}^{\prime}\right)+\sqrt{2 B_{\mathrm{tot}}^{2}\left(\nu_{c}^{\prime}\right)-1}\right]^{2} \\
& \times \frac{\Delta \nu_{c, \text { lossless }}}{\Delta \nu_{\mathrm{BW}, \text { lossless }}} .
\end{aligned}
$$

This equation together with Eq. (32) and (33) allows to determine the total transmission, reflection, and insertion loss at nonlinear resonance. The resonance shift $\Delta \nu_{c, 2 \mathrm{PA}}$ in the case of two-photon absorption can then be calculated by means of

$$
\Delta \nu_{c, 2 \mathrm{PA}} \approx\left|t_{\text {tot }}\left(\nu_{c}^{\prime}\right)\right|^{2} \Delta \nu_{c, \text { lossless }} .
$$

For a general frequency $\nu^{\prime} \neq \nu_{c}^{\prime}$, Eq. (37) and (39) are to be expanded to

$$
\begin{aligned}
& 1-B_{\mathrm{tot}}^{2}\left(\nu^{\prime}\right) \approx-\left|t_{\mathrm{tot}, \mathrm{NL}}\left(\nu^{\prime}\right)\right|^{4} \frac{c}{\pi \nu_{c}^{\prime}} \frac{\beta}{n_{2}} \frac{\Delta \nu_{c, \text { lossless }}}{\Delta \nu_{\mathrm{BW}, \text { lossless }}}, \\
& \Delta \nu_{\mathrm{NL}} \approx\left|t_{\mathrm{tot}, \mathrm{NL}}\left(\nu^{\prime}\right)\right|^{2} \Delta \nu_{c, \text { lossless }},
\end{aligned}
$$

which are the natural expansions of both Eq. (21) and (22). This also explains the $\left|t_{\text {tot,NL }}\left(\nu^{\prime}\right)\right|^{4}$, which is nothing more than the extension of the $\left|t_{\text {tot,NL }}\left(\nu^{\prime}\right)\right|^{2}$ dependence in the case of single-photon absorption to the two-photon case.

In contrast to Eq. (37) and (39), Eq. (31) —and thus Eqs. (32) and (33) -is not valid for general frequencies $\nu^{\prime} \neq \nu_{c}^{\prime}$. Instead, the following relation can be used: the nonlinear transmissivity $\left|t_{\text {tot,NL }}\left(\nu^{\prime}\right)\right|^{2}$ is approximately related to the linear transmissivity $\left|t_{\mathrm{tot}, \mathrm{L}}(\nu)\right|^{2}$, with $\nu^{\prime}=\nu+\left|t_{\mathrm{tot}, \mathrm{NL}}\left(\nu^{\prime}\right)\right|^{2}$ $\Delta \nu_{c, \text { lossless }}$ [see also Eq. (41) and compare with Sec. II], by 


$$
\frac{\left|t_{\mathrm{tot}, \mathrm{NL}}\left(\nu^{\prime}\right)\right|^{2}-\left|t_{\mathrm{tot}, \mathrm{L}}(\nu)\right|^{2}}{\left|t_{\mathrm{tot}, \mathrm{L}}(\nu)\right|^{2}}=V\left[1-B_{\mathrm{tot}}^{2}\left(\nu^{\prime}\right)\right],
$$

with $V$ a constant. This means that the relative change in the transmission at a certain frequency is proportional to the total insertion loss at that frequency. This is in agreement with what could be expected. To calculate the constant $V$, this equation is evaluated at $\nu=\nu_{c}^{\prime}$ or

$$
V=\frac{\left|t_{\mathrm{tot}}\left(\nu_{c}^{\prime}\right)\right|^{2}-1}{1-B_{\mathrm{tot}}^{2}\left(\nu_{c}^{\prime}\right)},
$$

so that

$$
\frac{\left|t_{\mathrm{tot}, \mathrm{NL}}\left(\nu^{\prime}\right)\right|^{2}-\left|t_{\mathrm{tot}, \mathrm{L}}\left(\nu^{\prime}\right)\right|^{2}}{\left|t_{\mathrm{tot}, \mathrm{L}}\left(\nu^{\prime}\right)\right|^{2}}=\left[\left|t_{\mathrm{tot}, \mathrm{NL}}\left(\nu_{c}^{\prime}\right)\right|^{2}-1\right] \frac{1-B_{\mathrm{tot}}^{2}\left(\nu^{\prime}\right)}{1-B_{\mathrm{tot}}^{\prime}\left(\nu_{c}^{\prime}\right)} .
$$

Using Eq. (41), this becomes

$$
\frac{\left|t_{\mathrm{tot}, \mathrm{NL}}\left(\nu^{\prime}\right)\right|^{2}}{\left|t_{\mathrm{tot}, \mathrm{L}}(\nu)\right|^{2}}-1=\left[\left|t_{\mathrm{tot}, \mathrm{NL}}\left(\nu_{c}^{\prime}\right)\right|^{2}-1\right] \frac{\left|t_{\mathrm{tot}, \mathrm{NL}}\left(\nu^{\prime}\right)\right|^{4}}{\left|t_{\mathrm{tot}, \mathrm{NL}}\left(\nu_{c}^{\prime}\right)\right|^{4}} \text {. }
$$

Finally, substituting Eq. (7), one obtains

$$
\begin{aligned}
\left|t_{\mathrm{tot}, \mathrm{NL}}\left(\nu^{\prime}\right)\right|^{6}-2 \frac{\nu^{\prime}-\nu_{c}}{\Delta \nu_{c, \text { lossless }}}\left|t_{\mathrm{tot}, \mathrm{NL}}\left(\nu^{\prime}\right)\right|^{4} \\
\quad+\left[\frac{\Delta \nu_{\mathrm{BW}, \text { lossless }}^{2}}{4 \Delta \nu_{c, \text { lossless }}^{2}}+\frac{\left(\nu^{\prime}-\nu_{c}\right)^{2}}{\Delta \nu_{c, \text { lossless }}^{2}}\right]\left|t_{\mathrm{tot}, \mathrm{NL}}\left(\nu^{\prime}\right)\right|^{2} \\
=\frac{\Delta \nu_{\mathrm{BW}, \text { lossless }}^{2}}{4 \Delta \nu_{c, \text { lossless }}^{2}}\left\{1-\left[1-\left|t_{\mathrm{tot}, \mathrm{NL}}\left(\nu_{c}^{\prime}\right)\right|^{2}\right] \frac{\left|t_{\mathrm{tot}, \mathrm{NL}}\left(\nu^{\prime}\right)\right|^{4}}{\left|t_{\mathrm{tot}, \mathrm{NL}}\left(\nu_{c}^{\prime}\right)\right|^{4}}\right\} .
\end{aligned}
$$

As can be seen in Fig. 4, the analytical results obtained by this equation show excellent agreement with the numerical results obtained by the nonlinear extension of CAMFR.

\section{EFFECT OF BOTH SINGLE- AND TWO-PHOTON ABSORPTIONS}

In most practical cases, both single- and two-photon absorption phenomena will be present-to a larger or lesser

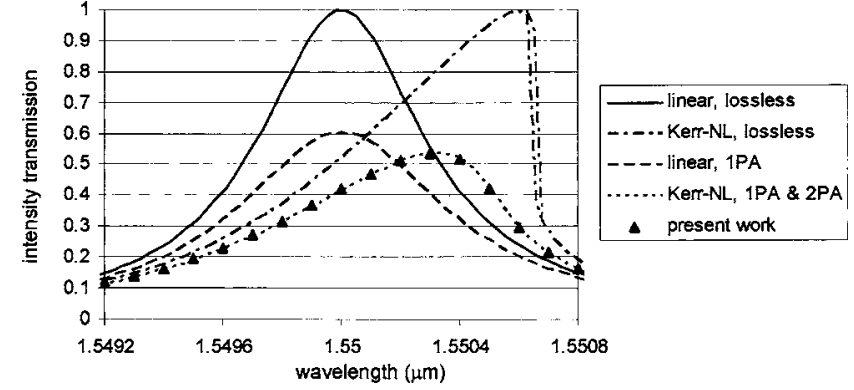

FIG. 6. Combined effect of single- and two-photon absorptions on the Kerrnonlinear resonator behavior. The results were obtained by simulation with the following parameters: $n_{a}=2.6, n_{b}=2.34, k_{a}=k_{b}=0.15 \times 10^{-3}, N_{\text {cav }}=1$, $N_{\mathrm{dbr}}=54$, and $n_{2}\left|E_{\mathrm{in}}\right|^{2}=\beta \lambda_{c}\left|E_{\mathrm{in}}\right|^{2}=8 \times 10^{-6}$. The triangles show the analytical results for the lossy, nonlinear situation obtained by means of Eq. (53).

extent. An example of such a situation is shown in Fig. 6. In this figure, the loss mechanisms of Fig. 2 and 4 are combined for the same input optical power. Both the linear and nonlinear transmission curves are shown in the case of lossless and lossy (due to 1PA and 2PA) structures.

In the figure, the combination of both loss effects can clearly be seen. The resonance shift $\Delta \nu_{c}$ in the lossy case is again much smaller than in the lossless case, but also smaller than the individual cases of Fig. 2 and 4. The lossy, nonlinear peak transmission is also smaller than the linear lossy one (which is due to 1PA, as discussed in Sec. III) like in the case of 2PA. And also the lossy, Kerr-nonlinear transmission relation has a different relative shape than its lossless counterpart.

To understand the Kerr-nonlinear behavior of a lossy resonator with both 1PA and 2PA effects, the results of Sec. III and IV must be combined. Based on Eqs. (21), (22), (37), and (41), the total insertion loss $1-C_{\text {tot }}^{2}(\nu)$ of the structure with $C_{\text {tot }}(\nu)$ the relative field amplitude after the complete resonator structure in the case of both 1PA and 2PA and the frequency shift $\Delta \nu_{\mathrm{NL}}$ at any frequency $\nu^{\prime}$ is straightforwardly given by

$$
1-C_{\mathrm{tot}}^{2}\left(\nu^{\prime}\right) \approx\left|t_{\mathrm{tot}, \mathrm{NL}}\left(\nu^{\prime}\right)\right|^{2}\left[\frac{\left(1-\left|r_{\mathrm{dbr}}\right|_{\nu_{c}}^{2} A_{\mathrm{dbr}}^{2} A_{\mathrm{cav}}^{2}\right)^{2}-\left|r_{\mathrm{dbr}}\right|_{\nu_{c}}^{2} A_{\mathrm{dbr}}^{2}\left(1-A_{\mathrm{dbr}}^{2} A_{\mathrm{cav}}^{2}\right)^{2}}{\left|t_{\mathrm{dbr}}\right|_{\nu_{c}}^{4} A_{\mathrm{dbr}}^{4} A_{\mathrm{cav}}^{2}}-1\right]-\left|t_{\mathrm{tot}, \mathrm{NL}}\left(\nu^{\prime}\right)\right|^{4} \frac{c}{\pi \nu_{c}^{\prime}} \frac{\beta}{n_{2}} \frac{\Delta \nu_{c, \text { lossless }}}{\Delta \nu_{\mathrm{BW}, \text { lossless }}},
$$

$$
\Delta \nu_{\mathrm{NL}} \approx\left|t_{\mathrm{tot}, \mathrm{NL}}\left(\nu^{\prime}\right)\right|^{2} \Delta \nu_{c, \text { lossless }} .
$$

Like in Sec. IV, the nonlinear transmission function $\left|t_{\text {tot,NL }}\left(\nu^{\prime}\right)\right|^{2}$ can, in good approximation, be related to the linear transmissivity $\left|t_{\mathrm{tot}, \mathrm{L}, 1 \mathrm{PA}}(\nu)\right|^{2}$, taking into account that the linear transmission now includes single-photon absorption with $\nu^{\prime}=\nu+\left|t_{\text {tot }}\left(\nu^{\prime}\right)\right|^{2} \Delta \nu_{c \text {,lossless }}$ [see Eq. (48) and compare with Sec. IV]:

$$
\frac{\left|t_{\mathrm{tot}, \mathrm{NL}}\left(\nu^{\prime}\right)\right|^{2}-\left|t_{\mathrm{tot}, \mathrm{L}, 1 \mathrm{PA}}(\nu)\right|^{2}}{\left|t_{\mathrm{tot}, \mathrm{L}, 1 \mathrm{PA}}(\nu)\right|^{2}}=W\left[1-C_{\mathrm{tot}}^{\prime 2}\left(\nu^{\prime}\right)\right],
$$

with $W$ a constant and $1-C_{\text {tot }}^{\prime 2}$ the 2PA loss part of Eq. (47). This means that the relative change in the transmission at a certain frequency (compared to the linear transmission which already includes $1 \mathrm{PA}$ ) is proportional to the total 2PA induced insertion loss at that frequency. This is again in agreement with what could be expected. To calculate the constant $W$, this equation is evaluated at $\nu^{\prime}=\nu_{c}^{\prime}$ [note that 
$\left.\left|t_{\mathrm{tot}, \mathrm{L}, 1 \mathrm{PA}}\left(\nu_{c}\right)\right|^{2}=\left|t_{\mathrm{tot}, \mathrm{NL}, 1 \mathrm{PA}}\left(\nu_{c}^{\prime}\right)\right|^{2}\right]$. In this way, one has

$$
W=\frac{\left|t_{\mathrm{tot}, \mathrm{NL}}\left(\nu_{c}^{\prime}\right)\right|^{2}-\left|t_{\mathrm{tot}, \mathrm{L}, 1 \mathrm{PA}}\left(\nu_{c}\right)\right|^{2}}{\left|t_{\mathrm{tot}, \mathrm{L}, 1 \mathrm{PA}}\left(\nu_{c}\right)\right|^{2}\left[1-C_{\mathrm{tot}}^{\prime 2}\left(\nu_{c}^{\prime}\right)\right]},
$$

so that

$$
\begin{aligned}
& \frac{\left|t_{\mathrm{tot}, \mathrm{NL}}\left(\nu^{\prime}\right)\right|^{2}-\left|t_{\mathrm{tot}, \mathrm{L}, 1 \mathrm{PA}}(\nu)\right|^{2}}{\left|t_{\mathrm{tot}, \mathrm{L}, 1 \mathrm{PA}}(\nu)\right|^{2}} \\
& =\left[\frac{\left|t_{\mathrm{tot}, \mathrm{NL}}\left(\nu_{c}^{\prime}\right)\right|^{2}-\left|t_{\mathrm{tot}, \mathrm{L}, 1 \mathrm{PA}}\left(\nu_{c}\right)\right|^{2}}{\left|t_{\mathrm{tot}, \mathrm{L}, 1 \mathrm{PA}}\left(\nu_{c}\right)\right|^{2}}\right] \frac{1-C_{\mathrm{tot}}^{\prime 2}\left(\nu^{\prime}\right)}{1-C_{\mathrm{tot}}^{\prime 2}\left(\nu_{c}^{\prime}\right)} .
\end{aligned}
$$

Using Eq. (48), this becomes

$$
\frac{\left|t_{\mathrm{tot}, \mathrm{NL}}\left(\nu^{\prime}\right)\right|^{2}}{\left|t_{\mathrm{tot}, \mathrm{L}, 1 \mathrm{PA}}(\nu)\right|^{2}}-1=\left[\frac{\left|t_{\mathrm{tot}, \mathrm{NL}}\left(\nu_{c}^{\prime}\right)\right|^{2}}{\left|t_{\mathrm{tot}, \mathrm{L}, 1 \mathrm{PA}}\left(\nu_{c}\right)\right|^{2}}-1\right] \frac{\left|t_{\mathrm{tot}, \mathrm{NL}}\left(\nu^{\prime}\right)\right|^{4}}{\left|t_{\mathrm{tot}, \mathrm{NL}}\left(\nu_{c}^{\prime}\right)\right|^{4}} .
$$

After substitution of Eq. (17), one obtains

$$
\begin{aligned}
& \left|t_{\mathrm{tot}, \mathrm{NL}}\left(\nu^{\prime}\right)\right|^{6}-\frac{2\left(\nu^{\prime}-\nu_{c}\right)}{\Delta \nu_{c, \text { lossless }}}\left|t_{\mathrm{tot}, \mathrm{NL}}\left(\nu^{\prime}\right)\right|^{4}+\left[\frac{\left(\nu^{\prime}-\nu_{c}\right)^{2}}{\Delta \nu_{c, \text { lossless }}^{2}}\right. \\
& \left.+\frac{\Delta \nu_{\mathrm{BW}}^{2}}{4\left|t_{\mathrm{tot}, \mathrm{L}, 1 \mathrm{PA}}\left(\nu_{c}\right)\right|^{2} \Delta \nu_{c, \text { lossless }}^{2}}\right]\left|t_{\mathrm{tot}, \mathrm{NL}}\left(\nu^{\prime}\right)\right|^{2} \\
& =\frac{\Delta \nu_{\mathrm{BW}}^{2}}{4 \Delta \nu_{c, \text { lossless }}^{2}}\left\{1-\left[1-\frac{\left|t_{\mathrm{tot}, \mathrm{NL}}\left(\nu_{c}\right)\right|^{2}}{\left|t_{\mathrm{tot}, \mathrm{L}, \mathrm{PA}}\left(\nu_{c}\right)\right|^{2}}\right] \frac{\left|t_{\mathrm{tot}, \mathrm{NL}}\left(\nu^{\prime}\right)\right|^{4}}{\left|t_{\mathrm{tot}, \mathrm{NL}}\left(\nu_{c}^{\prime}\right)\right|^{4}}\right\} .
\end{aligned}
$$

The transmissivity $\left|t_{\mathrm{tot}, \mathrm{L}, 1 \mathrm{PA}}\left(\nu_{c}\right)\right|^{2}$ in case of only 1PA has already been derived in Sec. IV. However, note that at this point, $\left|t_{\mathrm{tot}, \mathrm{NL}}\left(\nu_{c}^{\prime}\right)\right|^{2}$ is still unknown. Clearly, the total resonance transmission will be determined by both single- and two-photon processes. To determine $\left|t_{\mathrm{NL}, \text { tot }}\left(\nu_{c}^{\prime}\right)\right|^{2}$, simulation results obtained from the parameter space used in Sec. IV together with extinction coefficient variations of $K_{a}=(0-5)$ $\times 10^{-3}$ and $K_{b}=(0-5) \times 10^{-3}$ were polynomially fitted, leading to the following equation:

$$
\begin{aligned}
\mid t_{\mathrm{NL}, \mathrm{tot}} & \left.\left(\nu_{c}^{\prime}\right)\right|^{6}+\frac{1}{8}\left|t_{\mathrm{tot}, \mathrm{L}, 1 \mathrm{PA}}\left(\nu_{c}\right)\right|^{4}\left|t_{\mathrm{tot}, \mathrm{NL}, 2 \mathrm{PA}}\left(\nu_{c}^{\prime}\right)\right|^{2} \\
& +\frac{1}{8}\left|t_{\mathrm{tot}, \mathrm{NL}, 2 \mathrm{PA}}\left(\nu_{c}^{\prime}\right)\right|^{4}\left|t_{\mathrm{tot}, \mathrm{L}, 1 \mathrm{PA}}\left(\nu_{c}\right)\right|^{2} \\
& -\frac{1}{8}\left|t_{\mathrm{tot}, \mathrm{L}, \mathrm{PA} A}\left(\nu_{c}\right)\right|^{4}\left|t_{\mathrm{tot}, \mathrm{NL}}\left(\nu_{c}^{\prime}\right)\right|^{2} \\
& -\frac{1}{8}\left|t_{\mathrm{tot}, \mathrm{NL}, 2 \mathrm{PA}}\left(\nu_{c}^{\prime}\right)\right|^{4}\left|t_{\mathrm{tot}, \mathrm{NL}}\left(\nu_{c}^{\prime}\right)\right|^{2} \\
& -\frac{7}{8}\left|t_{\mathrm{tot}, \mathrm{NL}}\left(\nu_{c}^{\prime}\right)\right|^{4}\left|t_{\mathrm{tot}, \mathrm{L}, \mathrm{PAA}}\left(\nu_{c}\right)\right|^{2} \\
& -\frac{7}{8}\left|t_{\mathrm{tot}, \mathrm{NL}}\left(\nu_{c}^{\prime}\right)\right|^{4}\left|t_{\mathrm{tot}, \mathrm{NL}, 2 \mathrm{PA}}\left(\nu_{c}^{\prime}\right)\right|^{2} \\
& +\left|t_{\mathrm{tot}, \mathrm{L}, 1 \mathrm{PA}}\left(\nu_{c}\right)\right|^{2}\left|t_{\mathrm{tot}, \mathrm{NL}, 2 \mathrm{PA}}\left(\nu_{c}\right)\right|^{2}\left|t_{\mathrm{tot}, \mathrm{NL}}\left(\nu_{c}^{\prime}\right)\right|^{2} \\
& -\frac{1}{4}\left|t_{\mathrm{tot}, \mathrm{NL}}\left(\nu_{c}^{\prime}\right)\right|^{4}=0 .
\end{aligned}
$$

This surface represents a so called "saddle." A rms error of about 2\% was obtained with this fit. Note the $\left|t_{\text {tot,L,1PA }}\left(\nu_{c}\right)\right|^{2}-\left|t_{\text {tot,NL,2PA }}\left(\nu_{c}^{\prime}\right)\right|^{2}$ symmetry relation, which means that, e.g., $\left|t_{\mathrm{tot}, \mathrm{L}, 1 \mathrm{PA}}\left(\nu_{c}\right)\right|^{2}=0.7,\left|t_{\mathrm{tot}, \mathrm{NL}, 2 \mathrm{PA}}\left(\nu_{c}^{\prime}\right)\right|^{2}=0.2$, and $\left|t_{\text {tot,L,1PA }}\left(\nu_{c}\right)\right|^{2}=0.2,\left|t_{\text {tot,NL,2PA }}\left(\nu_{c}^{\prime}\right)\right|^{2}=0.7$, approximately result in the same total transmission. In this way, Eq. (53) is now fully determined.

\section{IMPACT OF LOSS MECHANISMS}

Resonator-based all-optical functionalities-such as alloptical switching-are typically related to the shift of the resonance frequency $\Delta \nu_{c^{1}}{ }^{1,2}$ As mentioned above, in the presence of single- and two-photon absorptions this resonance shift is, in good approximation, a factor $\left|t_{\mathrm{tot}, \mathrm{NL}}\left(\nu_{c}^{\prime}\right)\right|^{2}$ lower,

$$
\Delta \nu_{c, \text { lossy }} \approx\left|t_{\mathrm{tot}, \mathrm{NL}}\left(\nu_{c}^{\prime}\right)\right|^{2} \Delta \nu_{c, \text { lossless }} .
$$

Using Eqs. (34) and (35), the nonlinear figure of merit $(\mathrm{FOM}),{ }^{21} \mathrm{FOM} \equiv\left|n_{2} / \lambda \beta\right|$, can be written as

$$
\mathrm{FOM}=\frac{\Delta \phi_{c, \text { lossless }}}{2 \pi\left[1-B_{\text {tot, small }}\left(\nu_{c}^{\prime}\right)\right]} .
$$

With Eqs. (32), (37), and (55), this becomes

$$
\mathrm{FOM} \approx \frac{\left|t_{\mathrm{tot}, \mathrm{NL}, 2 \mathrm{PA}}\left(\nu_{c}^{\prime}\right)\right|^{3}}{2 \pi\left|t_{\mathrm{tot}, \mathrm{NL}}\left(\nu_{c}^{\prime}\right)\right|^{2}\left[1-\left|t_{\mathrm{tot}, \mathrm{NL}, 2 \mathrm{PA}}\left(\nu_{c}^{\prime}\right)\right|\right]} \frac{\Delta \nu_{c, \text { lossy }}}{\Delta \nu_{\mathrm{BW}}} .
$$

Note, however, that $\Delta \nu_{\mathrm{BW}}$ is the lossless resonator bandwidth. As mentioned above, the shape of the transmission relation changes when loss is introduced. $\Delta \nu_{\mathrm{BW} \text {,lossy }}$ can approximately be calculated by solving Eq. (53). In this way, one obtains, for the lossy bandwidth in presence of both single-photon and two-photon absorptions,

$\Delta \nu_{\mathrm{BW}, \text { lossy }} \approx \sqrt{\frac{3\left|t_{\mathrm{tot}, \mathrm{L}, \mathrm{PA}}\left(\nu_{c}\right)\right|^{2}-\mid t_{\mathrm{tot}, \mathrm{NL}}\left(\nu_{c}^{\prime}\right)^{2}}{2\left|t_{\mathrm{tot}, \mathrm{NL}}\left(\nu_{c}^{\prime}\right)\right|^{2}\left|t_{\mathrm{tot}, \mathrm{L}, 1 \mathrm{PA}}\left(\nu_{c}\right)\right|^{2}}} \Delta \nu_{\mathrm{BW}, \text { lossless }}$,

so that

$$
\begin{aligned}
\mathrm{FOM} \approx & \frac{\left|t_{\mathrm{tot}, \mathrm{NL}, 2 \mathrm{PA}}\left(\nu_{c}^{\prime}\right)\right|^{3} \sqrt{3\left|t_{\mathrm{tot}, \mathrm{L}, 1 \mathrm{PA}}\left(\nu_{c}\right)\right|^{2}-\left|t_{\mathrm{tot}, \mathrm{NL}}\left(\nu_{c}^{\prime}\right)\right|^{2}}}{2 \sqrt{2} \pi\left|t_{\mathrm{tot}, \mathrm{NL}}\left(\nu_{c}^{\prime}\right)\right|^{3}\left|t_{\mathrm{tot}, \mathrm{L}, \mathrm{PA}}\left(\nu_{c}\right)\right|\left[1-\left|t_{\mathrm{tot}, \mathrm{NL}, 2 \mathrm{PA}}\left(\nu_{c}^{\prime}\right)\right|\right]} \\
& \times \frac{\Delta \nu_{c, \text { lossy }}}{\Delta \nu_{\mathrm{BW}, \text { lossy }}} .
\end{aligned}
$$

Two of the most interesting semiconductor materials for ultrafast, Kerr-nonlinear operation are the AlGaAs and $\mathrm{Si}$ systems. Around the telecom wavelength of $\lambda=1.55 \mu \mathrm{m}$, they have a nonlinear figure of merit value of

$$
\begin{aligned}
& \mathrm{FOM}_{\mathrm{AlGaAs}} \approx 5.38, \\
& \mathrm{FOM}_{\mathrm{Si}} \approx 0.37,
\end{aligned}
$$

where the values of the nonlinear coefficients were used as reported in Refs. 18 and 22. To evaluate the potential to these materials for Kerr-nonlinear resonator-based functionalities, the effect of two-photon absorption should be acceptable: in most cases, the main linear loss mechanisms are related to fabrication. The impact of 2PA can be expressed by the relative transmission parameter $\left|t_{\text {tot,NL }}\left(\nu_{c}^{\prime}\right)\right|^{2} /\left|t_{\mathrm{tot}, \mathrm{L}, 1 \mathrm{PA}}\left(\nu_{c}\right)\right|^{2}$. In the lossless case, bistability is obtained for a resonance shift of $\Delta \nu_{c}=(4 \sqrt{3} / 9) \Delta \nu_{\mathrm{BW}}$ (see Sec. II). In the lossy case, the calculation is much more complex, however, in good approximation, one again obtains $\Delta \nu_{c \text {,lossy }} \approx(4 \sqrt{3 / 9}) \Delta \nu_{\mathrm{BW}, \text { lossy }}$. Using Eq. (59), $\left|t_{\text {tot,NL }}\left(\nu_{c}^{\prime}\right)\right|^{2} /\left|t_{\text {tot,L, } 1 \text { PA }}\left(\nu_{c}\right)\right|^{2}$ can be calculated for this resonance shift as function of $\left|t_{\mathrm{tot}, \mathrm{L}, 1 \mathrm{PA}}\left(\nu_{c}\right)\right|^{2}$ for dif- 


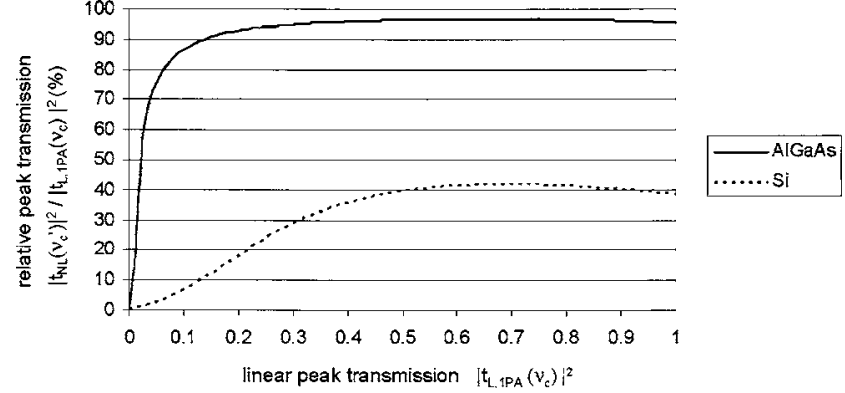

FIG. 7. Remaining nonlinear transmission as a function of the linear transmission for both $\mathrm{AlGaAs}$ and $\mathrm{Si}$ for a resonance shift of $\Delta \lambda_{c}$ $=(4 \sqrt{3} / 9) \Delta \lambda_{\text {BW }}$.

ferent nonlinear materials. The result for $\mathrm{AlGaAs}$ and $\mathrm{Si}$ are shown in Fig. 7.

As can be seen, the effect of $2 \mathrm{PA}$ is quite severe in the case of $\mathrm{Si}$ and is only acceptable for very low linear losses. For the AlGaAs system on the other hand, the impact of 2PA is very limited, even for low linear transmissions. The total rms error on this curve is approximately $5 \%$.

For a linear peak transmission of $\left|t_{\mathrm{tot}, \mathrm{L}, 1 \mathrm{PA}}\left(\nu_{c}\right)\right|^{2}=0.5$, the two-photon impact is visualized in Fig. 8 and 9.

The remaining peak transmission is in the AlGaAs system about $48 \%$, while in the case of Si, only $20 \%$ transmission remains for the maximum stable resonance shift of $\Delta \nu_{c, \text { lossy }}=(4 \sqrt{3} / 9) \Delta \nu_{\mathrm{BW}, \text { lossy }}$. In addition, the bandwidth increases approximately $4 \%$ in the $\mathrm{AlGaAs}$ case, while for $\mathrm{Si}$, this increase is already a factor of 2 .

\section{CONCLUSIONS}

Using analytical approximations, a Kerr-nonlinear model was derived which takes into account both linear and twophoton absorption effects. This model has been used to evaluate the impact of two-photon absorption for different nonlinear material systems at the telecom wavelength $\lambda$ $=1.55 \mu \mathrm{m}$. For the AlGaAs system, the effect is almost neg-

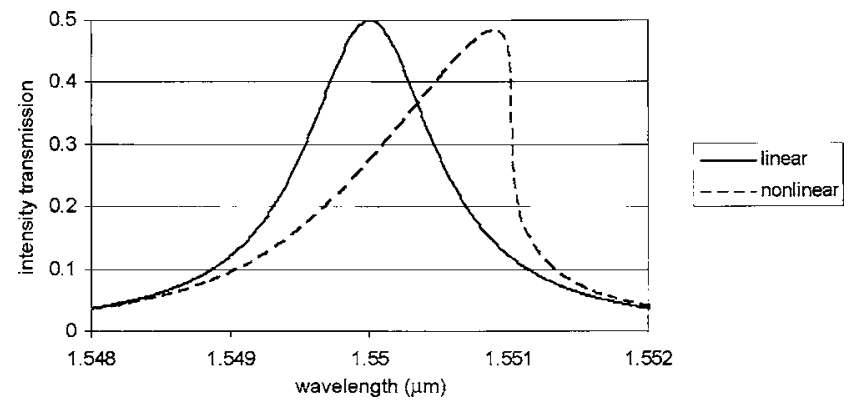

FIG. 8. Example of linear and nonlinear transmission curves of an AlGaAs material system with $\left|t_{\mathrm{tot}, \mathrm{L}, 1 \mathrm{PA}}\left(\nu_{c}\right)\right|^{2}=0.5$. The nonlinear curve corresponds to a resonance shift of $\Delta \nu_{c, \text { lossy }}=(4 \sqrt{3} / 9) \Delta \nu_{\mathrm{BW}, \text { lossy }}$.

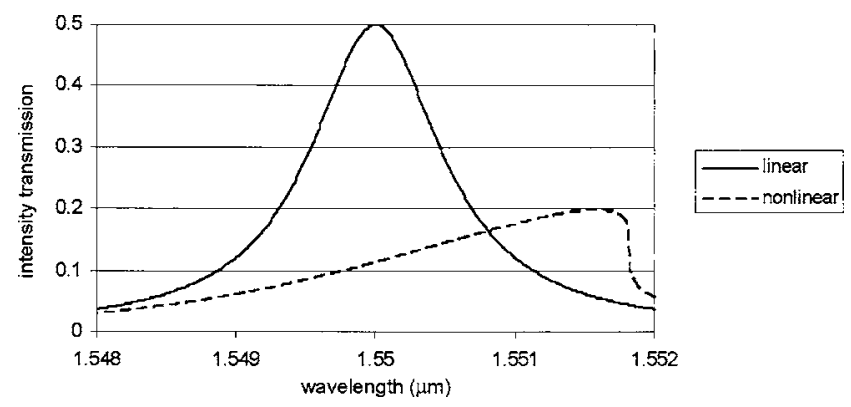

FIG. 9. Example of linear and nonlinear transmission curves of a Si material system with $\left|t_{\text {tot,L,1PA }}\left(\nu_{c}\right)\right|^{2}=0.5$. The nonlinear curve corresponds to resonance shift of $\Delta \nu_{c, \text { lossy }}=(4 \sqrt{3} / 9) \Delta \nu_{\mathrm{BW}, \text { lossy }}$.

ligible and linear (fabrication) loss will be dominant. For Si, however, two-photon absorption can result in severe degradation and is only acceptable in the case of low linear losses.

\section{ACKNOWLEDGMENTS}

Part of this work was performed in the context of the Belgian BELSPO IAP-PHOTON project. One of the authors (G.P.) thanks the Flemish Fund for Scientific Research (FWO-Vlaanderen) for a doctoral fellowship. Another author (P.B.) thanks the Flemish Fund for Scientific Research (FWO-Vlaanderen) for a postdoctoral fellowship.

${ }^{1}$ G. Priem, I. Notebaert, P. Bienstman, G. Morthier, and R. Baets, J. Appl. Phys. 97, 023104 (2005).

${ }^{2}$ G. Priem, I. Notebaert, B. Maes, P. Bienstman, G. Morthier, and R. Baets, IEEE J. Sel. Top. Quantum Electron. 10, 1070 (2004).

${ }^{3}$ J. E. Heebner and R. B. Boyd, Opt. Lett. 24, 847 (1999).

${ }^{4}$ S. Blair, Opt. Lett. 27, 613 (2002).

${ }^{5}$ Y. Chen and S. Blair, Opt. Express 12, 3353 (2004).

${ }^{6}$ Y. Chen, G. Pasrija, B. Farhang-Boroujeny, and S. Blair, Opt. Lett. 28, 1945 (2003).

${ }^{7}$ A. Melloni, F. Morichetti, and M. Martinelli, Opt. Quantum Electron. 35, 365 (2003).

${ }^{8}$ M. Soljačić, S. G. Johnson, S. H. Fan, M. Ibanescu, E. Ippen, and J. D. Joannopoulos, J. Opt. Soc. Am. B 19, 2052 (2002).

${ }^{9}$ D. Pelinovsky, J. Sears, L. Brzozowski, and E. H. Sargent, J. Opt. Soc. Am. B 19, 43 (2002).

${ }^{10}$ J. He and M. Cada, IEEE J. Quantum Electron. 27, 1182 (1991).

${ }^{11}$ U. Peschel, T. Peschel, and F. Lederer, IEEE J. Quantum Electron. 30, 1241 (1994).

${ }^{12}$ S. Radic, N. George, and G. Agrawal, J. Opt. Soc. Am. B 12, 671 (1995).

${ }^{13}$ J. Lee and G. P. Agrawal, IEEE J. Quantum Electron. 39, pp. 508-515 (2003).

${ }^{14}$ D. Weaire and J. P. Kermode, J. Opt. Soc. Am. B 3, 1706 (1986).

${ }^{15}$ P. W. Smith and E. H. Turner, Appl. Phys. Lett. 30, 280 (1977).

${ }^{16}$ S. Blair, J. E. Heebner, and R. B. Boyd, Opt. Lett. 27, 357 (2002).

${ }^{17}$ M. Soljačić, M. Ibanescu, S. G. Johnson, Y. Fink, and J. D. Joannopoulos, Phys. Rev. E 66, 055601 (2002).

${ }^{18}$ A. Villeneuve, C. Yang, C. Lin, and H. Lin, Appl. Phys. Lett. 62, 2465 (1993).

${ }^{19}$ P. Bienstman and R. Baets, Opt. Quantum Electron. 33, 327 (2001).

${ }^{20}$ B. Maes, P. Bienstman, and R. Baets, Opt. Quantum Electron. 36, 15 (2004).

${ }^{21}$ K. W. Delong and G. I. Stegeman, Appl. Phys. Lett. 57, 2063 (1990).

${ }^{22}$ M. Dinu, F. Quochi, and H. Garcia, Appl. Phys. Lett. 82, 954 (2003). 